\title{
Laparoscopic Restorative Proctocolectomy in Adenomatus Patients
}

\author{
Fabio Guilherme Campos ${ }^{1^{*}}$ \\ ${ }^{1}$ Gastroenterology Department, Colorectal Unit, Hospital das Clinicas, Medical School, University of Sao Paulo, Sao Paulo, Brazil
}

\section{A R T I C L E I N F O}

Article type:

Letter to Editor

Article history:

Received: 01 Dec 2011

Revised: 30 Jan 2012

Accepted:14 Feb 2012

\section{Keywords}

Laparoscopy

Proctocolectomy, Restorative

Adenomatous Polyposis Coli

Iliostomy

\section{Dear Editor,}

I read with great interest the series presented by Bananzadeh et al. (1). This series includes a group of 19 patients who underwent laparoscopic restorative proctocolectomy (RPC) without ileostomy, performed by the same surgeon, to treat Familial Adenomatous Polyposis (FAP) between October 2008 and May 2011. Ileal pouch-anal anastomosis (IPAA) is currently the standard surgical alternative for the majority of ulcerative colitis (UC) and FAP patients. Despite the complexity of the operation, IPAA is safe (mortality: $0.5-1 \%$ ) and carries an acceptable risk of non-life-threatening complications (10-25\%), achieving good long-term functional outcomes with excellent patient satisfaction (over 95\%). During the last decade, the surgical technique has evolved significantly, mainly due to the growing incorporation of laparoscopic approaches. Because it is a complex technical procedure, a temporary ileostomy proximal to the ileal pouch has typically been performed (2). Thus, the most controversial aspect of the study discussed here being the omission of ileostomy in a series of laparoscopic surgeries. A protective ileostomy may reduce anastomosis leakage, prevent pelvic sepsis and fistulization, thus preserv-

\footnotetext{
* Corresponding author: Fabio Guilherme Campos, Gastroenterology Department, Colorectal Unit, Hospital das Clinicas, Medical School, University of Sao Paulo, Sao Paulo, Brazil.E-mail: fgmcampos@terra.com.br

DOI:10.5812/jmiss.3677

Copyright (C) 2012, Minimally Invasive Surgery Research Center and Mediterranean \& Middle Eastern Endoscopic Surgery Association. This is an open-acces article distributed under the terms of the Creative Commons Attribution License, which permits unrestricted use, distribution, and reproduction in any medium, provided the original work is properly cited.
}

Please cite this paper as:

Campos FG. Laparoscopic Restorative Proctocolectomy in Adenomatus Patients. J Minim Invasive Surg Sci. 2012;1(2): 80-2. DOI: $10.5812 /$ jmiss.3677

ing pouch function. Consequently, it should also prevent the need for re-laparotomy and most importantly, pouch failure. The rationale for this decision is based on the fact that a protective ileostomy may limit the severity of septic complications, as the prevalence of pouch-related septic complications varies between $6 \%$ and $37 \%$ (2). Furthermore, most patients are able to accept this temporary stoma well, although it may be a source of complications after its construction or closure. These complications may include dehydration and metabolic disorders, peristomal irritation, anastomotic fistula, intestinal obstruction, and others (3).

Although a protective ileostomy is still performed in the vast majority of series, its omission is associated with a similar rate of septic complications and may also provide economic advantages for select patients. By avoiding an ileostomy, the surgeon should prevent potential associated problems such as high output and complications of the stoma and its closure. Selection criteria for this choice should exclude clinical factors (high doses of steroids, malnutrition, toxicity or anemia) and technical factors (difficult procedures with intraoperative complications). Furthermore, surgeons must be sure that the ileoanal anastomosis is tension-free, that it is supplied with adequate blood flow, that the tissue rings are intact and that there are no air leaks $(3,4)$. Within this context, a German group studied 706 consecutive patients (494 UC, $212 \mathrm{FAP}$ ) in an attempt to identify subgroups of patients who were at high risk for pouch-relat- 
ed sepsis (2). As in previous reports, they found that the risk of pouch sepsis was markedly greater in patients with UC than in patients with FAP, indicating the predominant role of the underlying disease in the development of infection. Moreover, patients with FAP presented higher risk only if anastomotic tension had occurred (RR3.60, $P=0.0086)$ and if they were older than 50 years of age $(P=0.004)(2)$. The first series of IAA without diverting ileostomy was reported in 1990, in a small series of UC patients. Since then, other publications have reported their results after IAA without ileostomy for UC and FAP patients, showing rates of pelvic sepsis that varied from $4 \%$ to $22 \%$.

In a Mayo Clinic study, Galandiuk et al. (5) compared 37 patients without ileostomy to a matched group with ileostomy operated on during the same period (1981 to 1990). They reported that 8 patients (22\%) without ileostomy and four patients (11\%) with ileostomy experienced one or more postoperative pouch-related complications. Complications requiring reoperation in UC and FAP patients without ileostomy occurred more frequently in patients either taking steroids or having had previous pelvic radiation therapy. They concluded that J-pouch construction with IPAA can be safely performed without diverting ileostomy provided some selection factors (absolute lack of tension on the anastomosis, good blood supply to the terminal ileum, good general health and no recent intake of steroids at the time of surgery) are taken into account. In a randomized study, rates of pelvic sepsis were similar between patients with (22 cases) or without (23 cases) ileostomy (6). There were only two ileoanal anastomotic leaks, one in each group. Furthermore, loop ileostomy was associated with a high incidence of complications (52\%). These results indicate that the low risk of pelvic sepsis is not increased by omitting a protective ileostomy. In the Mount Sinai series with UC and FAP patients (7), the authors observed that the rate of IPAA suture line dehiscence was not significantly different between the two groups (ileostomy, 4/69 (6\%), vs. no ileostomy, 6/74 $(8 \%) ; P>0.05)$, even in patients submitted to mucosectomy, which may increase the procedure's rate of morbidity. RPC without ileostomy results in significantly fewer episodes of intestinal obstruction, fewer instances of re-exploration and fewer total days in the hospital. The group from the Saint Antoine Hospital in Paris (8) reported their experience with 84 FAP and UC patients who underwent IPAA without ileostomy between 1993 and 1998. Early and late complications were seen in 25 (30\%) and 23 patients (27\%) respectively, requiring reoperation in 13 , including three temporary ileostomies and one pouch excision for Crohn's disease. Five patients (5.9\%) developed an early septic complication of the pelvis. Morbidity and functional results were equivalent to those obtained with a defunctioning ileostomy. Based on those results, they stated that for a selected group of patients undergoing an IAA, a defunctioning ileostomy could be avoided. More recently, a study from the Cleveland Clinic showed that the omission of ileostomy may even provide cost savings in terms of the whole treatment (9).
In an attempt to better identify patients who may be candidates for ileostomy omission during RPC, the group from St Marks Hospital in London reviewed 4013 operations performed between 1977 and 2005 (10). Proximal diversion was performed in 3196 of 3733 patients (85.6\%). With the help of logistic regression analysis, the independent factors favoring omission of ileostomy were: stapled anastomosis (odds ratio [OR], 6.4), no preoperative corticosteroid use (OR, 3.2), familial adenomatous polyposis diagnosis (OR, 2.6), cancer diagnosis (OR, 3.4), female gender $(\mathrm{OR}, 1.6)$ and age at surgery younger than $26(\mathrm{OR}, 2.1)(P<0.01$ for all). Omission of proximal diversion demonstrated no significant effect on postoperative adverse events, although it was associated with a 2-day increase in the median length of hospital stay $(P<0.01)$. However, its safety is controversial. While there are articles showing that the omission of temporary ileal diversion has a relatively low complication rate and provides excellent fecal control, others state that RPC without diversion is not as safe as RPC with diversion, especially in patients taking more than $20 \mathrm{mg}$ of prednisone/day (11). In other UC patient series, one-stage restorative proctocolectomy without a defunctioning ileostomy was associated with an increased risk of death, which is the reason its routine use should not be recommended (12).

In another recent paper from Saint Antoine Hospital (13), the authors reported their experience with 71 patients (38 females) who underwent laparoscopic RPC between November 2004 and February 2010. Indications were FAP (34), UC (35), indeterminate colitis (1) and Lynch syndrome (1). Laparoscopic RPC was performed as a one-stage procedure in 49 patients, and after a sub-total colectomy in 22. Seven patients in each group underwent the formation of a diverting stoma. Sixteen patients experienced at least one postoperative complication. The postoperative morbidity was $29 \%(n=4 / 14)$ and $21 \%(n=12 / 21)$ in patients with and without a stoma $(P=0.8)$, and the rate of fistula was $21 \%$ and $5 \%$, respectively $(P=0.08)$. Seven percent of patients with a stoma and $16 \%$ without a stoma had intra-abdominal fluid collection $(P=0.7)$. Nine patients required reoperation, which was not influenced by the presence or absence of a diverting stoma. The results of this study are similar those of other laparoscopic RPC series.

Thus, the data from the literature presented here support the idea that an ileostomy may be safely omitted in select patients, especially FAP. First, IPAA complications are generally less common in FAP than in UC (14). At diagnosis, FAP patients usually present with few symptoms and good general condition, a different picture from those suffering from UC. And when comparing septic complications with and without ileostomy, most cases were attributed to steroid use (11). The French group from Saint Antoine reported a $4 \%$ rate of septic complications in FAP compared with $6 \%$ in UC in two series of unselected consecutive patients undergoing IPAA with ileostomy (16). Other comparative studies have also shown higher rates of septic complications without ileostomy, but the risk of secondary ileostomy has 
remained below $6 \%$. However, it is important to note that revision surgery may be necessary only in cases of disseminated peritoneal infection, and less severe cases may be controlled with antibiotics. In the series reported by Cohen et al.(15), $18 \%$ of the 71 patients without ileostomy developed an anastomotic fistula, but a temporary ileostomy was only required in one (1.4\%).

The risk of developing postoperative fertility problems after complications from RPC should not be used to contraindicate procedures without ileostomy. Currently it is well recognized that the risk of fertility problems is not associated with the type of surgery, indication for surgery, complications or other comorbid conditions. Postoperative fertility problems are more common among women who had their first surgical procedure at a younger age (16). The omission of ileostomy may have a great impact on young patients, who usually place a high value on bodily appearance. While large-bowel techniques are evolving rapidly, the selection criteria for omitting an ileostomy after laparoscopic RPC, especially in FAP, still remain to be clarified. Lopez-Rosales et al. (17) reported good results in eight out of 10 patients who underwent IPAA without protection. Ky et al. (18) registered 11 postoperative complications and three reoperations among 32 one-stage RPC. In our own series, one patient subjected to a one-stage procedure developed a postoperative fistula that was successfully treated with intestinal deviation (19). So far, we have preferred to perform laparoscopic RPC with ileostomy, due to the potential risk of desmoid tumors in FAP, which has been associated with surgical trauma, among other predictive factors.

In this article, Bananzadeh et al. (1) described the treatment of 19 patients with a mean age of 34 years (range 22-40 years ) who experienced no anastomotic leakage after two weeks post-operation. As hospitalization length after surgery varied from 4 to 7 days, the authors were only able to report complications such as copious diarrhea in 6 patients (31\%), and transitory fecal incontinence in 2 patients (10\%). However, complications from RPC are usually late and a longer followup is necessary in this group. By initiating a discussion about the avoidance of ileostomy after RPC, their article achieved their main purpose regarding this important issue. A review of the pertinent literature leads to the conclusion that selective omission of a protective ileostomy may be safe and is associated with similar septic complications and failure rates when compared with stoma patients. However, this finding leads us to critically evaluate FAP patient selection criteria, in which an experienced surgical team, a patient with a good clinical status and a procedure without adverse intraoperative outcomes should be considered.

\section{Authors' Contribution}

F.G.C contributed $100 \%$ to prepare this article.

\section{Financial disclosure}

None declared.

\section{References}

1. Bananzadeh A, Rezaianzadeh A, Ghahramani L, Hosseini SV. Laparoscopic Restorative Proctocolectomy Without Diverting Loop Ileostomy in Patients With Familial Adenomatous Polyposis. J Minim Invasive Surg Sci. 2012;1(1):21-3.

2. Heuschen UA, Hinz U, Allemeyer EH, Autschbach F, Stern J, Lucas M, et al. Risk factors for ileoanal J pouch-related septic complications in ulcerative colitis and familial adenomatous polyposis. Ann Surg. 2002;235(2):207-16

3. Banasiewicz T, Marciniak R, Kaczmarek E, Krokowicz P, Paszkowski J, Lozynska-Nelke A, et al. The prognosis of clinical course and the analysis of the frequency of the inflammation and dysplasia in the intestinal J-pouch at the patients after restorative proctocolectomy due to FAP. Int J Colorectal Dis. 2011;26(9):1197-203.

4. Remzi FH, Fazio VW, Gorgun E, Ooi BS, Hammel J, Preen M, et al. The Outcome After Restorative Proctocolectomy With or Without Defunctioning Ileostomy. Diseases of the Colon \& Rectum. 2006;49(4):470-7,10.1007/s10350-006-0509-2.

5. Galandiuk S, Wolff BG, Dozois RR, Beart RW, Jr. Ileal pouch-anal anastomosis without ileostomy. Dis Colon Rectum.1991;34(10):870-3.

6. Grobler SP, Hosie KB, Keighley MR. Randomized trial of loop ileostomy in restorative proctocolectomy. BrJ Surg. 1992;79(9):903-6.

7. Gorfine SR, Gelernt IM, Bauer JJ, Harris MT, Kreel I. Restorative proctocolectomy without diverting ileostomy. Dis Colon Rectum. 1995;38(2):188-94.

8. Gignoux BM, Dehni N, Parc R, Tiret E. [Ileal pouch anal-anastomosis without protective ileostomy]. Gastroenterol Clin Biol.2002;26(8-9):671-4.

9. Joyce MR, Kiran RP, Remzi FH, Church J, Fazio VW. In a select group of patients meeting strict clinical criteria and undergoing ileal pouchanal anastomosis, the omission of a diverting ileostomy offers cost savings to the hospital. Dis Colon Rectum. 2010;53(6):905-10.

10. Lovegrove RE, Tilney HS, Remzi FH, Nicholls RJ, Fazio VW, Tekkis PP. To divert or not to divert: A retrospective analysis of variables that influence ileostomy omission in ileal pouch surgery. Arch Surg. 2011;146(1):82-8.

11. Tjandra JJ, Fazio VW, Milsom JW, Lavery IC, Oakley JR, Fabre JM. Omission of temporary diversion in restorative proctocolectomy-is it safe? Dis Colon Rectum.1993;36(11):1007-14.

12. Williamson ME, Lewis WG, Sagar PM, Holdsworth PJ, Johnston D. One-stage restorative proctocolectomy without temporary ileostomy for ulcerative colitis: a note of caution. Dis Colon Rectum. 1997;40(9):1019-22.

13. Hor T, Zalinski S, Lefevre JH, Shields C, Attal E, Tiret E, et al. Feasibility of laparoscopic restorative proctocolectomy without diverting stoma. Dig Liver Dis. 2012;44(2):118-22.

14. Kartheuser A, Stangherlin P, Brandt D, Remue C, Sempoux C. Restorative proctocolectomy and ileal pouch-anal anastomosis for familial adenomatous polyposis revisited. Fam Cancer. 2006;5(3):241-60; discussion 61-2.

15. Cohen Z, McLeod RS, Stephen W, Stern HS, O'Connor B, Reznick R. Continuing evolution of the pelvic pouch procedure. Ann Surg. 1992;216(4):506-11; discussion 11-2.

16. Nieuwenhuis MH, Douma KF, Bleiker EM, Bemelman WA, Aaronson NK, Vasen HF. Female fertility after colorectal surgery for familial adenomatous polyposis: a nationwide cross-sectional study. Ann Surg. 2010;252(2):341-4.

17. Lopez-Rosales F, Gonzalez-Contreras Q, Muro LJ, Berber MM, de Leon HT, Fernandez OV, et al. Laparoscopic total proctocolectomy with ileal pouch anal anastomosis for ulcerative colitis and familial adenomatous polyposis: initial experience in Mexico. Surg Endosc. 2007;21(12):2304-7.

18. Ky AJ, Sonoda T, Milsom JW. One-stage laparoscopic restorative proctocolectomy: an alternative to the conventional approach? Dis Colon Rectum. 2002;45(2):207-10; discussion 10-1.

19. Campos FG, Araujo SE, Melani AG, Pandini LC, Nahas SC, Cecconello I. Surgical outcomes of laparoscopic colorectal resections for familial adenomatous polyposis. Surg Laparosc Endosc Percutan Tech. 2011;21(5):327-33. 